\title{
78 Potential muscle stressors
}

Most muscles are designed to work in functional pairs. When a muscle is flexed by the agonist, its counterpart or antagonist can counter act this flexion by extending the muscle again. The local force exerted by the neuromuscular system depends on the number, cross-section and structure of the individual muscle fibers. Optimal coordination of the respective cooperating muscles and the efficient provision of energy also play an important role.

Muscles can be exerted in a great multitude of different ways. For example, during the development of dynamic forces within a sequence of movements, the emphasis is on shortening of the muscles, also known as concentric contraction (i.e., muscle contraction in which the ends of the muscle move together towards a common center). When the application of force against resistance lengthens the muscle, e. g. like the braking force necessary to walk downhill or the body bracing itself after raising a weight, this is known as eccentric work (elongation of the fibers back out from a common center). If the contraction is directed against insurmountable resistance, this is a static strength exercise. During this isometric muscle tensioning, muscle length remains unchanged. A muscle's maximum eccentric strength is always greater than its dynamic maximum strength, and, depending on training status, 5-40\% greater than its static strength.

In terms of sport or exercise, we are usually dealing with dynamic forces. The sequence of movements during running, for example, but also in many other sports, is characterized by rhythmic alternation between concentric contraction and eccentric braking force. The contraction velocity dictates the performance of the related muscles.

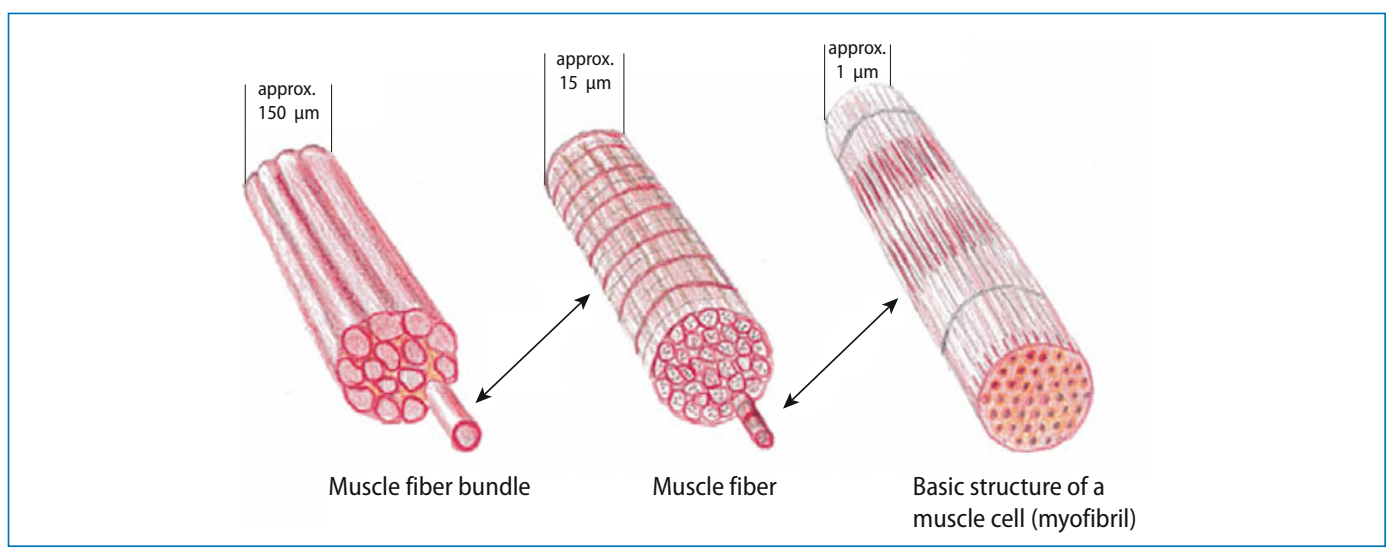

Fig. 78.1 Structure of fasciated muscle

D. Mathias, Staying healthy from 1 to 100, DOI 10.1007/978-3-662-49195-9_78,

(C) Springer-Verlag Berlin Heidelberg 2016 